\title{
A COMUNICAÇÃO NA PROMOÇÃO DA DIGNIDADE EM CUIDADOS PALIATIVOS: DESAFIOS PARA A ENFERMAGEM
}

\author{
COMMUNICATION IN PROMOTING DIGNITY IN \\ PALLIATIVE CARE: CHALLENGES FOR NURSING
}

\section{COMUNICACIÓN EN LA PROMOCIÓN DE LA DIGNIDAD EN LOS CUIDADOS PALIATIVOS: DESAFIOS PARA LA ENFERMERÍA}

\author{
Bruno Miguel Freire Monho ${ }^{1}$ \\ Inês Margarida Peralta Ferreira ${ }^{2}$ \\ Mariana Ferreira Bernardino Ribeiro ${ }^{3}$ \\ Tânia Sofia Cardoso Alves ${ }^{4}$ \\ Maria Deolinda Antunes da Luz Lopes Dias Maurício
}

\begin{abstract}
Como citar este artigo: Monho BMF, Ferreira IMP, Ribeiro MFB, Alves TSC, Maurício MDALPD. A comunicação na promoção da dignidade em cuidados paliativos: desafios para a enfermagem. Rev baiana enferm. 2021;35:e34788.

Objetivo: compreender a influência da comunicação enquanto instrumento básico de Enfermagem na promoção da dignidade em Cuidados Paliativos. Método: reflexão teórica mediante revisão da literatura e com recurso ao Modelo da Dignidade de Chochinov. A pesquisa de artigos científicos foi realizada na biblioteca do conhecimento online, na base de dados das publicações Elsevier e na plataforma agregadora de bases de dados EBSCO Host Web, publicados durante o período 2010-2016. Resultados: identificou-se intervenções associadas às competências comunicacionais dos enfermeiros que promovem a dignidade dos clientes em cuidados paliativos. As intervenções comunicacionais que mais promovem a dignidade são as que transmitem ao cliente paliativo o respeito pela sua individualidade e valorização da sua história pessoal. Conclusão: o enfermeiro deve adotar uma postura de compreensão empática, escuta ativa, disponibilidade, atenção às necessidades emocionais, aos componentes não verbais (como a presença física e o contacto visual), à gestão de expectativas e o incentivo do autocuidado.
\end{abstract}

Descritores: Enfermagem. Cuidados Paliativos. Comunicação. Dignidade Humana.

Objective: to understand the influence of communication as a basic nursing instrument in the promotion of dignity in Palliative Care. Method: theoretical reflection through literature review and using Chochinov's Dignity Model. The research of scientific articles was carried out on the online knowledge library, on the Elsevier publications database and on the database-aggregating platform EBSCO Host Web, published during the period 2010-2016. Results: interventions associated with nurses' communication skills that promote the dignity of clients in palliative care were identified. The communication interventions that most promote dignity are those that transmit to the palliative client

Enfermeiro. Enfermeiro de Cuidados Gerais na Unidade de Cuidados Paliativos da Casa de Saúde da Idanha. Belas, Portugal. https://orcid.org/0000-0003-0787-036X. Enfermeira. Enfermeira de Cuidados Gerais no Serviço de Medicina do Hospital Prof. Dr. Fernando Fonseca. Amadora, Portugal. https://orcid.org/0000-0003-0 I061940.

3 Enfermeira. Enfermeira de Cuidados Gerais no Serviço de Cirurgia do Hospital Prof. Dr. Fernando Fonseca. Amadora, Portugal. https://orcid.org/0000-0002-68I I5352.

4 Enfermeira. Pesquisadora independente. Lisboa, Portugal. https://orcid.org/0000-0003-0852-1460.

5 Professora do Ensino Superior. Doutora em Psicologia da Saude. Professora Coordenadora na Escola Superior de Enfermagem de Lisboa. Lisboa, Portugal. dluz@esel.pt. http://orcid.org/0000-0002-6565-1694. 
respect for their individuality and appreciation of their personal history. Conclusion: nurses should adopt a posture of empathic understanding, active listening, availability, attention to emotional needs, nonverbal components (such as physical presence and visual contact), management of expectations and encouragement of self-care.

Descriptors: Nursing. Palliative Care. Communication. Human Dignity.

Objetivo: entender la influencia de la comunicación como instrumento básico de enfermería en la promoción de la dignidad en los Cuidados Paliativos. Método: reflexión teórica a través de la revisión de la literatura y el uso del Modelo de Dignidad de Chochinov. La investigación de artículos científicos se llevó a cabo en la biblioteca de conocimientos en linea, en la base de datos de publicaciones Elsevier y en la plataforma agregadora de bases de datos EBSCO Host Web, publicados durante el periodo 2010-2016. Resultados: se identificaron intervenciones asociadas a las habilidades de comunicación de las enfermeras que promueven la dignidad de los clientes en los cuidados paliativos. Las intervenciones de comunicación que más promueven la dignidad son las que transmiten al cliente paliativo el respeto por su individualidad y apreciación de su bistoria personal. Conclusión: las enfermeras deben adoptar una postura empática de comprensión, escucha activa, disponibilidad, atención a las necesidades emocionales, componentes no verbales (como la presencia física y el contacto visual), gestión de expectativas y fomento del autocuidado.

Descriptores: Enfermería. Cuidados Paliativos. Comunicación. Dignidad Humana.

\section{Introdução}

Perante a confrontação do Enfermeiro com o sofrimento do outro e o contacto com o seu lado mais frágil e vulnerável em Cuidados Paliativos (CP), surgiu a necessidade de focalizar a presente análise crítica e reflexiva na promoção da dignidade nesse contexto. A pertinência desta abordagem é corroborada pelos códigos de ética e deontologia profissionais que afirmam que as intervenções de enfermagem são realizadas com a preocupação da defesa da dignidade da pessoa humana ${ }^{(1)}$. O ser humano é um ser de relação. Desde os seus primórdios que o Homem é considerado um ser social e de relações interpessoais. Como tal, a comunicação torna-se um processo fulcral para que consigamos viver em comunidade.

Transpondo essa realidade para a Enfermagem, a comunicação afigura-se como um dos seus instrumentos básicos. Assim sendo, "[...] o enfermeiro deve garantir o sucesso da comunicação que utiliza no âmbito da prestação de cuidados, uma vez que níveis de comunicação eficazes conduzem a resultados mais positivos" (2:32). Dessa forma, a importância da comunicação é tanto maior quanto a constatação de que comunicar com o Outro constitui uma das nossas principais atividades. Baseado em nossa experiência empírica na área, infere-se que a comunicação é um fator determinante na promoção da dignidade em CP, sendo um aspeto preponderante na promoção da dignidade humana ${ }^{(3)}$.

O objectivo deste estudo é compreender a influência da comunicação enquanto instrumento básico de Enfermagem na promoção da dignidade em Cuidados Paliativos.

\section{Método}

Este trabalho consiste numa análise crítica e reflexiva de uma extensa revisão da literatura sobre uma temática específica e delimitada.

Foi efetuada uma pesquisa bibliográfica em bases de dados tendo em vista a credibilidade e a atualidade da investigação produzida, especialmente para a área da Enfermagem. Nesta investigação, optou-se por realizar a pesquisa de artigos científicos em língua portuguesa e inglesa, na biblioteca do conhecimento online (b-on), na base de dados das publicações Elsevier, e na plataforma agregadora de bases de dados EBSCO Host Web (recorrendo essencialmente à MEDLINE with full text, CINAHL with full text e Cochrane Database of Systematic Reviews), publicados durante o período 2010-2016. Após a análise dos textos, foi elaborado o relatório final de curso "O resultado 
espelha uma aliança entre a análise crítica do panorama atual e a análise dos textos". A análise crítica parte da experiência empírica que os elementos do grupo possuem na área, bem como a formação básica em CP. O presente estudo decorreu entre março e junho de 2016.

\section{Resultados e Discussão}

\section{Cuidados paliativos}

O adjetivo e substantivo paliativo vem de "paliado (particípio de paliar) sob a forma radical paliat- + -ivo, da forma histórica palliatîvo, paleativo, paliativo", com origem no latim para expressar "cobrir com uma capa; encobrir, disfarçar". O adjetivo paliativo é atualmente atribuído ao que "tem a qualidade de acalmar, de abrandar temporariamente um mal (diz-se de medicamento ou tratamento)", ao que "serve para atenuar um mal ou protelar uma crise (diz-se de meio, iniciativa, etc.), ${ }^{(4)}$. Decorrente dessa ideia, os CP podem definir-se como cuidados totais aos clientes portadores de doenças que apresentem risco de vida, tal como às suas famílias, prestados por uma equipa multidisciplinar, no período temporal em que a doença já não responde com eficácia aos tratamentos curativos $^{(5)}$. Esta definição pode ainda ser enriquecida com a da Organização Mundial de Saúde (OMS): "[...] uma abordagem que visa melhorar a qualidade de vida dos doentes - e suas famílias - que enfrentam problemas decorrentes de uma doença incurável e/ou grave e com prognóstico limitado"(6:2). Estes afirmam, ainda, que a abordagem se centra na "[...] prevenção e alívio do sofrimento, com recurso à identificação precoce e tratamento rigoroso dos problemas não só físicos como a dor, mas também dos psicossociais e espirituais" ${ }^{\text {(6:2) }}$.

Assim, os CP representam o cuidado holístico dos clientes com doenças progressivas e avançadas que não apresentam resposta ao tratamento curativo. Os CP contemplam quatro pilares fundamentais: controlo sintomático - mobilização de estratégias farmacológicas e não farmacológicas; comunicação adequada - utilizar competências relacionais e comunicacionais eficazes para com os clientes e famílias; apoio à família - incluir a família no processo de cuidados e avaliar as suas necessidades; trabalho em equipa - integrar os contributos dos profissionais das várias áreas, com o intuito comum do conforto e bem-estar dos clientes e famílias ${ }^{(6)}$. Essas quatro vertentes são encaradas com igual importância, sendo que a prática de $\mathrm{CP}$ com qualidade não seria concretizável se alguma delas fosse menosprezada ${ }^{(6)}$.

\section{Enfermagem em Cuidados Paliativos - Conceptualização Atual}

$\mathrm{Na}$ era pós-moderna surgiram as primeiras teorias de Enfermagem que consideravam a adequação dos princípios holísticos às práticas de cuidados $^{(7)}$. É nessa nova corrente de pensamento que surge o paradigma da transformação, representando uma mudança da mentalidade cuja dinâmica emana da interação contínua entre fenómenos complexos e globais com o mundo ${ }^{(7)}$. Para a Enfermagem, o foco de atenção passou a ser a individualização do cuidado humano e a especificidade de cada pessoa enquanto ser único. Essa escola de pensamento em Enfermagem compreende a Teoria da Humanização do Cuidado de Jean Watson, na qual a pessoa é vista como uma harmonia entre todas as suas vertentes: corpo, alma, espírito e saúde. O cuidar em Enfermagem não se manifesta apenas numa atitude ou numa emoção; corresponde sim, a um processo intersubjetivo, um ideal moral, que tem como objetivo proteger, melhorar e preservar a dignidade da pessoa cuidada ${ }^{(8)}$.

Em CP, este conceito de cuidar reflete-se quando os enfermeiros interagem com os clientes, para compreenderem as suas experiências e possibilitarem a expressão de sentimentos e pensamentos. Assim, estabelece-se como objetivo diminuir o sofrimento dos clientes e famílias, de modo a facilitar o seu processo de adaptação à experiência que vivem ${ }^{(6)}$. Duas das dimensões mais importantes destes cuidados, centradas na relação de ajuda, são a empatia e o respeito pela dignidade de cada cliente e família. 
Assim, mediante a utilização de diferentes estratégias comunicativas por parte dos enfermeiros, promove-se o princípio da autonomia e ajuda os clientes e famílias a refletirem sobre a tomada de decisões mais adequadas, tendo em conta os seus valores, as suas necessidades e o seu estado emocional $^{(7)}$.

\section{Dignidade}

A dignidade é um conceito complexo e de difícil definição, uma vez que os seus contornos se alteram consoante os contextos em que é abordada $^{(9)}$. Na Roma Antiga, em pleno séc. XIII, a expressão Dignitas significava "mérito", "reputação", estando associada à noção de hierarquia $^{(9)}$. Um ponto crucial na transição para os conceitos modernos de dignidade surge no Renascimento, quando, em 1480, o filósofo italiano Pico della Mirandola (1463-1494) associa o conceito da dignidade humana à liberdade ${ }^{(10)}$.

Anos mais tarde, inserido no contexto dos edificadores ideológicos do liberalismo, é o filósofo alemão Immanuel Kant ${ }^{(11)}$ (1724-1804), quem dá uma das contribuições mais decisivas para a definição deste conceito, ao afirmar que "No reino dos fins, tudo tem um preço ou uma dignidade. Quando uma coisa tem um preço, pode pôr-se em vez dela qualquer outra coisa como equivalente; mas quando uma coisa está acima de todo o preço, e [...] não permite equivalente, então ela tem dignidade" ${ }^{(11: 77)}$. O homem não tem preço, não tem equivalente, tem, sim, dignidade.

Surge então a premissa "Age de tal modo que trates a humanidade, tanto na tua pessoa como na do outro, sempre e ao mesmo tempo, como um fim e nunca simplesmente como um meio"(12:8).
Com o fim do Holocausto tornou-se imperativo construir um mundo sob novos alicerces ideológicos. Para tal, foi elaborada, em 1948, a Declaração Universal dos Direitos Humanos ${ }^{(13)}$. No documento constam 30 artigos, que nomeiam os direitos humanos básicos, assentes na premissa de que a dignidade humana resulta do reconhecimento de um valor, traduzindo-se como o princípio moral fundamentado na finalidade do homem e não na sua utilização enquanto um meio. São as manifestações de racionalidade, liberdade e de finalidade em si mesmos que tornam o homem um ser em constante desenvolvimento, numa busca incessante da realização pessoal.

\section{Dignidade em Cuidados Paliativos}

Como já foi referido, em cuidados paliativos, dada a vulnerabilidade dos seus doentes, torna-se fundamental que a abordagem dos profissionais de saúde tenha como um dos seus focos a preservação da dignidade dos doentes. O princípio da dignidade em ética médica expressa-se no desenvolvimento de cuidados paliativos ${ }^{(14)}$. Pretendem providenciar condições adequadas ao estatuto de ser humano, tanto em vida como durante o processo de morte ${ }^{(14)}$.

De acordo com esta última ideia, a "Pessoa" deve ser vista como um ser multidimensional que transcende os limites corpóreos. Na tentativa de esquematizar os diferentes domínios da dignidade em CP, um dos mais reconhecidos trabalhos desenvolvidos na evidência científica é o Modelo da Dignidade em Doentes em Fim de $\mathrm{Vida}^{(15)}$. O objetivo deste modelo teórico centra-se na identificação das variáveis clínico-demográficas que influenciam a dignidade (Quadro 1). 
Quadro 1 - Categorias, temas, subtemas e itens do Modelo da Dignidade de Doentes em Fim de Vida

\begin{tabular}{|c|c|c|}
\hline $\begin{array}{l}\text { Preocupações Relacionadas } \\
\text { com a Doença }\end{array}$ & Recursos Pessoais de Dignidade & $\begin{array}{c}\text { Recursos Sociais de } \\
\text { Dignidade }\end{array}$ \\
\hline \multirow[t]{2}{*}{$\begin{array}{l}\text { Nível de independência: } \\
\text { Acuidade cognitiva } \\
\text { Autonomia funcional } \\
\text { Descompensação sintomática: } \\
\text { Distress físico } \\
\text { Distress psicológico: } \\
\text { Incerteza clínica } \\
\text { Ansiedade/medo da morte }\end{array}$} & $\begin{array}{l}\text { Perspetivas protetoras da dignidade: } \\
\text { Continuidade do self } \\
\text { Preservação de papéis } \\
\text { Legado/herança } \\
\text { Manutenção do orgulho } \\
\text { Esperança } \\
\text { Autonomia/controlo } \\
\text { Aceitação } \\
\text { Resiliência }\end{array}$ & \multirow[t]{2}{*}{$\begin{array}{l}\text { Privacidade (e os seus } \\
\text { limites) } \\
\text { Suporte social } \\
\text { Tonalidade do Cuidar } \\
\text { Sobrecarga dos } \\
\text { Cuidadores } \\
\text { Preocupações com o } \\
\text { futuro }\end{array}$} \\
\hline & $\begin{array}{l}\text { Práticas protetoras da dignidade: } \\
\text { Viver o momento } \\
\text { Manter a normalidade } \\
\text { Procura do conforto espiritual }\end{array}$ & \\
\hline
\end{tabular}

Fonte: Chochinov HM, Hack T, McClement S, Kristjanson L, Harlos $\mathrm{M}^{(15)}$.

Dessa forma, surge no discurso dos doentes uma vertente relacional da dignidade, que se constrói na interação com o Outro ${ }^{(6)}$. Consequentemente, a comunicação torna-se um aspeto essencial na promoção da dignidade das pessoas doentes.

\section{Comunicação em cuidados paliativos}

Ao longo dos tempos, foram muitos os autores que escreveram sobre a comunicação, o seu conceito e as suas características enquanto instrumento básico de enfermagem. Neste caso, importa-nos definir o conceito aplicado à área de Enfermagem. Assim, a comunicação pode definir-se como " um processo de criação e de recriação de informação, de troca, de partilha e de colocar em comum sentimentos e emoções entre pessoas"(16:23).

Estudo (16) afirma que "A comunicação se transmite de maneira consciente ou inconsciente pelo comportamento verbal e não verbal, e de modo mais global, pela maneira de agir dos intervenientes"(16:23). Assim, a comunicação permite-nos “ [...] aprender e [...] compreender as intenções, as opiniões, os sentimentos e as emoções sentidas pela outra pessoa e, segundo o caso, criar laços significativos com ela"(16:23).

Importa ainda referir que as componentes da comunicação não se restringem à troca verbal de informação, de conteúdo cognitivo, pois existe igualmente uma contrapartida afetiva manifestada no comportamento não verbal e no modo de ser da pessoa. De igual modo, é possível constatar que aproximadamente $75 \%$ da comunicação nas práticas de Enfermagem é não verbal, sendo estes mais autênticos e genuínos e menos sujeitos à reprovação do consciente ${ }^{(6)}$. Apesar de estas manifestações poderem ser menos evidentes, não deixam de ser menos importantes que a comunicação verbal, visto que são as emoções subentendidas nas palavras expressadas pela pessoa que fazem o outro reagir ${ }^{(16)}$.

A literatura revela que a mobilização de competências comunicacionais é uma intervenção terapêutica eficaz, proporcionando à pessoa "[...] compartilhar medos, receios, dúvidas e sofrimento, contribuindo para a diminuição do stress psicológico e garantindo a sua autonomia, proporcionando mais qualidade e conquistando mais satisfação pessoal"(17:11). 
Por essa razão, comunicar com eficácia assume-se como uma necessidade básica na intervenção ao cliente/família em CP. Comunicar eficazmente, nesse contexto, constitui-se um desafio, uma vez que exige a mobilização e o desenvolvimento de perícias básicas para a comunicação entre a tríade enfermeiro-cliente-família, emergindo assim a necessidade de uma formação adequada ${ }^{(6,18)}$.

\section{A influência da Comunicação na promoção da Dignidade Humana}

Muitos profissionais têm levado a cabo investigações com o intuito de explorar o significado de morrer com dignidade e os aspetos que a influenciam em CP. Um dos aspetos essenciais com um forte impacto na preservação da dignidade é a comunicação estabelecida entre os profissionais de saúde e as pessoas doentes ${ }^{(3)}$. Surge então a necessidade de compreender que o enfermeiro pode pôr em prática intervenções comunicacionais para salvaguardar a dignidade das pessoas de quem cuida. Na tentativa de fazer emergir tais intervenções comunicacionais, fez-se confluir o Modelo da Dignidade de Doentes em Fim de $\mathrm{Vida}^{(15)}$ com as competências comunicacionais de um enfermeiro de cuidados gerais. Apresentamos, por sequência, os Quadros 2, 3 e 4 resultantes das reflexões realizadas pelos autores e conseguintes.

Quadro 2 - Intervenções de Enfermagem na dimensão das preocupações relacionadas com a doença

\begin{tabular}{|l|l|l|}
\hline \multicolumn{1}{|l|}{ Preocupações Relacionadas com a Doença } & \multicolumn{1}{|c|}{ Intervenções de Enfermagem } \\
\hline $\begin{array}{l}\text { Nivel de } \\
\text { independência }\end{array}$ & $\begin{array}{l}\text { Acuidade cognitiva } \\
\text { Autonomia funcional }\end{array}$ & $\begin{array}{l}\text { Educar os clientes sobre o que é normativo. } \\
\text { Orientar os clientes para a realidade, quando } \\
\text { apropriado. }\end{array}$ \\
\hline $\begin{array}{l}\text { Descompensação } \\
\text { sintomática }\end{array}$ & $\begin{array}{l}\text { Distress físico } \\
\text { Distress psicológico }\end{array}$ & $\begin{array}{l}\text { Aliar estratégias farmacológicas a estratégias } \\
\text { comunicacionais (manter o contacto visual, estar } \\
\text { presente, fazer recurso do toque terapêutico). }\end{array}$ \\
\cline { 3 - 4 } & $\begin{array}{l}\text { Dialogar sobre a morte e os medos dos clientes, } \\
\text { recorrendo à escuta activa das suas percepções. } \\
\text { Partilhar experiências, quando solicitado. } \\
\text { Na transmissão de más notícias, o enfermeiro } \\
\text { deve enfatizar o potencial terapêutico dos clientes, } \\
\text { informando-os do que pode ser feito. }\end{array}$ \\
\hline
\end{tabular}

Fonte: Elaboração própria.

No que concerne ao distress físico, ao aliar estratégias comunicacionais a estratégias farmacológicas quando ocorre uma descompensação sintomática, o enfermeiro potencia o conforto do cliente, auxiliando-o a lidar com este momento $^{(18)}$. Ao aplicar as intervenções do Quadro 2, o enfermeiro estará a potenciar a preparação mental dos clientes e famílias para a evolução da doença, antecipando possíveis perdas funcionais e cognitivas. Além disso, permite que os clientes iniciem um processo de luto preparatório, isto é, o processo de aceitação da sua finitude, sem perder a fé e a esperança ${ }^{(18)}$. Estudo ${ }^{(19)}$ demonstra que a maior parte dos clientes desejam que alguém que cuidou de pessoas em fim de vida um médico ou enfermeiro paliativista - partilhe experiências que os ajudem a clarificar a evolução da doença, atribuindo-lhes um significado. 
Quadro 3 - Intervenções de Enfermagem na dimensão dos recursos pessoais da dignidade

\begin{tabular}{|c|c|c|}
\hline \multicolumn{2}{|c|}{ Recursos Pessoais da Dignidade } & \multirow{8}{*}{$\begin{array}{l}\text { Intervenções de Enfermagem } \\
\text { Incentivar o estabelecimento de metas realistas. } \\
\text { Promover a partilha de emoções e sentimentos, } \\
\text { dando resposta a possíveis necessidades espirituais. } \\
\text { Transmitir informação verdadeira. } \\
\text { Incentivar a preservação de hábitos e rotinas } \\
\text { pessoais. } \\
\text { Estimular os clientes a agir da forma com que mais } \\
\text { se identificam. } \\
\text { Incentivar os clientes a focarem-se no presente. } \\
\text { Negociar com os clientes a possibilidade de } \\
\text { resolver problemas antigos. }\end{array}$} \\
\hline \multirow{8}{*}{$\begin{array}{l}\text { Perspetivas } \\
\text { protetoras da } \\
\text { dignidade }\end{array}$} & Continuidade do self & \\
\hline & Preservação de papéis & \\
\hline & Legado/herança & \\
\hline & Manutenção do orgulho & \\
\hline & Esperança & \\
\hline & Autonomia/controlo & \\
\hline & Aceitação & \\
\hline & Resiliência & \\
\hline \multirow{3}{*}{$\begin{array}{l}\text { Práticas } \\
\text { protetoras da } \\
\text { dignidade }\end{array}$} & Viver o momento & \\
\hline & Manter a normalidade & \\
\hline & $\begin{array}{l}\text { Procura do conforto } \\
\text { espiritual }\end{array}$ & \\
\hline
\end{tabular}

Fonte: Elaboração própria.

Estudo $^{(6,20)}$ diz que na constante tentativa de defender a dignidade dos seus clientes, o enfermeiro deve: dar atenção às suas necessidades emocionais, bem como às das famílias; negociar e estabelecer metas realistas, deixando certo que existirão "dias bons e dias maus"; discutir a vivência de um dia de cada vez, focando-se no presente - firmando esta crença na premissa carpe diem -; e equilibrar a transmissão de informação verdadeira com a promoção de esperança ${ }^{(6,20)}$. Seguindo essa linha de pensamento, estas estratégias que facilitam a vivência do processo de doença em fase paliativa, ainda incluem: incentivar os clientes a aproveitar os dias em que se sintam bem e auxiliá-los a ultrapassar os dias em que se sentem pior; não antecipar frequentemente cenários negativos que poderão não ocorrer; incentivá-los a manter hábitos e rotinas que vão ao encontro dos seus gostos e preferências (como, por exemplo, respeitar os horários do cliente), numa tentativa de preservar um senso de normalidade às pessoas cuidadas; incentivar os clientes a não ficarem focados na morte ininterruptamente, porque "[...] a vida não para quando se está a morrer" (6:361).

Quadro 4 - Intervenções de Enfermagem na dimensão dos recursos sociais de dignidade

\begin{tabular}{|l|l|}
\hline $\begin{array}{l}\text { Recursos Sociais de } \\
\text { Dignidade }\end{array}$ & \multicolumn{1}{c|}{ Intervenções de Enfermagem } \\
\hline Suporte social & Promover a preservação de relações significativas. \\
\hline Tonalidade do Cuidar & $\begin{array}{l}\text { Adotar uma postura de apoio, firmada no afeto, respeito e } \\
\text { compreensão empática. } \\
\text { Estabelecer interações genuínas e autênticas. } \\
\text { Utilizar o humor quando adequado (evitando situações de crise como a } \\
\text { descompensação sintomática, por exemplo). }\end{array}$ \\
\hline
\end{tabular}

Fonte: Elaboração própria. 
Vários são os estudos que revelam que o estabelecimento de relações significativas com as pessoas que nos rodeiam é um aspeto primordial na promoção da dignidade em $\mathrm{CP}^{(3)}$. Para que tal seja possível, é fundamental que o enfermeiro escute os clientes de forma ativa, transmitindo-lhes confiança e uma compreensão empática genuína. Além disso, não é possível construir relações significativas se nelas não estiver presente o afeto e o amor, num processo de feeback positivo(3). Assim sendo, torna-se basal que os enfermeiros adquiram e aprimorem competências comunicacionais e relacionais que permitam a construção de relações promotoras da dignidade.

A dignidade é um conceito com limites ténues e de difícil definição. Espera-se que este trabalho tenha contribuído para uma melhor compreensão do que é dignidade, ajudando a perspetivá-la nos cuidados de Enfermagem. Dessa forma, pretende-se sensibilizar a comunidade de Enfermagem para a adoção de uma prática clínica que salvaguarde a dignidade dos clientes, potenciando-a nesta fase de vulnerabilidade.

\section{Conclusão}

Vivenciar o declínio cognitivo e funcional do outro, culminando com a sua morte, obriga $\mathrm{O}$ enfermeiro a pensar e confrontar-se com a sua própria morte, o que inevitavelmente traz uma componente emocional associada. Aqui, o investimento no autoconhecimento do enfermeiro e o desenvolvimento de competências comunicacionais e relacionais tornam-se imprescindiveis. Ter consciência das suas aptidões e limitações é uma ferramenta importante para a construção de uma relação de ajuda terapêutica eficaz, dado que o enfermeiro consegue ver até onde a sua intervenção produz o efeito pretendido, promovendo o bem-estar físico, psicológico e emocional de quem está a cuidar. Como tal, a importância do autoconhecimento reside na forma como passamos a nos encarar e a encarar o mundo que nos rodeia.
Do trabalho realizado, podemos afirmar que a dignidade é um valor intrínseco do ser humano, decorrente do seu reconhecimento enquanto Pessoa. Assentes na premissa de que o Homem é um ser de relação, em constante comunicação com quem o rodeia.

De forma a operacionalizar a promoção da dignidade em CP, propõe-se que o enfermeiro adote uma postura de compreensão empática, firmada no respeito pela pessoa que cuida. Além disso, o enfermeiro dispõe de um leque de intervenções comunicacionais à sua disposição, dentro do qual se encontram: a resposta às necessidades emocionais, pela transmissão de informação verdadeira; a gestão de expectativas, que permite a manutenção da esperança pela definição de metas realistas; e o incentivo ao autocuidado, que promove a autonomia do cliente mediante a valorização do seu projeto pessoal.

\section{Colaborações:}

1 - Concepção, projeto, análise e interpretação dos dados: Bruno Miguel Freire Monho, Inês Margarida Peralta Ferreira, Mariana Ferreira Bernardino Ribeiro, Tânia Sofia Cardoso Alves e Maria Deolinda Antunes da Luz Lopes Dias Maurício;

2 - Redação do artigo e revisão crítica relevante do conteúdo intelectual: Bruno Miguel Freire Monho e Maria Deolinda Antunes da Luz Lopes Dias Maurício;

3 - Aprovação final da versão a ser publicada: Maria Deolinda Antunes da Luz Lopes Dias Maurício.

\section{Referências}

1. Nunes L, Amaral M, Gonçalves R, coordenadores. Código Deontológico do Enfermeiro: dos Comentários à Análise dos Casos. Lisboa (PT): Ordem dos Enfermeiros; 2005.

2. Coelho MTV, Sequeira C. Comunicação terapêutica em enfermagem: como a caraterizam os enfermeiros. Rev port enferm saúde mental [Internet]. 2014 jun [cited 2018 Dec 2];(11):31-8. 
Available from: http://www.scielo.mec.pt/pdf/ rpesm/n11/n11a05.pdf

3. Guo Q, Jacelon CS. An Integrative Review of Dignity in End-Of-Life Care. Palliative Med. 2014;28(7):931-40. DOI: 10.1177/0269216314528399

4. Houaiss A, Villar M, Franco FMM. Dicionário eletrônico Houaiss da língua portuguesa. Rio de Janeiro: Objetiva; 2001.

5. Twycross R. Cuidados Paliativos. 2a ed. Tradução de Almeida JN. Lisboa (PT): Climepsi Editores; 2005.

6. Barbosa A, Galiriça Neto I, coordenadores. Manual de Cuidados Paliativos. 2a ed. Lisboa (PT): Núcleo de Cuidados Paliativos do Centro de Bioética da Faculdade de Medicina da Universidade de Lisboa; 2010.

7. Sapeta P. Cuidar em Fim de Vida: o Processo de Interacção Enfermeiro-Cliente. Loures (PT): Lusociência; 2011.

8. Watson J. Enfermagem: Ciência Humana e Cuidar. Uma Teoria de Enfermagem. Tradução de Enes J. Loures (PT): Lusociência; 2002.

9. Brennan F. Dignity: A unifying concept for Palliative Care and human rights. Prog Palliat Care. 2014;22(2):88-96. DOI: 10.1179/ 1743291X13Y.0000000064

10. Otero P. Instituições Políticas e Constitucionais. Coimbra (PT): Edições Almedina; 2009.

11. Kant I. Fundamentação da Metafísica dos Costumes. Lisboa (PT): Edições 70; 1991.

12. Conselho Nacional de Ética para as Ciências da Vida. Reflexão Ética sobre a dignidade Humana [Internet]. Lisboa (PT); 1999. (Documento de trabalho no 26/CNECV/99) [cited 2019 Oct 8]. Available from: http://bibliobase.sermais.
pt:8008/BiblioNET/Upload/PDF16/012478\%20 CNECV\%20reflex\%C3\%A30\%20\%C3\%A9tica \%2026.CNECV.99.pdf

13. United Nations Human Rights. Office of the High Commissioner. Declaração Universal dos Direitos Humanos [Internet]. Geneva (CH); 1948 [cited 2019 Oct 8]. Available from: https://www.ohchr. org/EN/UDHR/Pages/Language.aspx?LangID=por

14. Hottois G, Missa JN. Nova enciclopédia da bioética: medicina, ambiente tecnologia. Lisboa (PT): Instituto Piaget; 2003.

15. Chochinov HM, Hack T, McClement S, Kristjanson L, Harlos M. Dignity in the terminally ill: a developing empirical model. Soc Sci Med. 2002;54(3):433-43. DOI: 10.1016/s0277-9536(01)00084-3

16. Phaneuf M. Comunicação, entrevista, relação de ajuda e validação. Loures (PT): Lusociência; 2005.

17. Pereira LPS. A Comunicação na Humanização dos Cuidados Paliativos [tese na Internet]. Porto (PT): Universidade do Porto; 2014 [cited 2016 Apr 1]. Available from: https://repositorio-aberto.up.pt/ bitstream/10216/78541/2/34633.pdf

18. Östlund, U, Brown H, Johnston B. Dignity conserving care at end-of-life: A narrative review. Eur J Oncol Nurs. 2012;16(4):353-67. DOI: 10.1016/j.ejon.2011.07.010

19. Grant E, Murray SA, Kendall M, Boyd K, Tilley S, Ryan D. Spiritual Issues and Needs: Perspectives From Patients With Advanced Cancer and Nonmalignant Disease. A Qualitative Study. Palliat Support Care. 2004;2(4):371-8. DOI: $10.1017 /$ s1478951504040490

Recebido: 4 de dezembro de 2019

Aprovado: 4 de março de 2020

Publicado: 26 de novembro de 2020

A Revista Baiana de Enfermagem utiliza a Licença Creative Commons - Atribuição-NãoComercial 4.0 Internacional. https://creativecommons.org/licenses/by-nc/4.0/

Este artigo é de acesso aberto distribuído sob os termos da Licença Creative Commons (CC BY-NC). Esta licença permite que outros remixem, adaptem e criem a partir do seu trabalho para fins não comerciais. Embora os novos trabalhos tenham de lhe atribuir o devido crédito e não possam ser usados para fins comerciais, os usuários não têm de licenciar esses trabalhos derivados sob os mesmos termos. 